\title{
THE ALGEBRAIC DECAY OF EQUATORIAL ROSSBY WAVES IN A SHEAR FLOW
}

\author{
JOHN P. BOYD and ZAPHIRIS D. CHRISTIDIS \\ Department of Atmospheric and Oceanic Science, University of Michigan, \\ 2455 Hayward Avenue, Ann Arbor, MI 48109 (U.S.A.)
}

(Received July 24, 1986; revised May 21, 1987; accepted May 27, 1987)

\section{ABSTRACT}

Boyd, J.P. and Christidis, Z.D., 1987. The algebraic decay of equatorial Rossby waves in a shear flow. Dyn. Atmos. Oceans, 11: 139-151.

Through numerical integration, we show that equatorial Rossby waves, like their midlatitude counterparts, decay algebraically in the limit $t \rightarrow \infty$ in a linear shear flow. For small times, the growth expected for some components does not translate into any growth of the wave disturbance as a whole when the initial condition has a broad Fourier spectrum. The conclusion is that Rossby waves will amplify with time only when the mean flow has an inflection point or when the initial eddy field is strongly concentrated in long waves tilted against the shear.

\section{INTRODUCTION}

Midlatitude Rossby waves in a linear shear flow decay algebraically with time, perhaps after a temporary episode of growth. Orr's (1907) analytic solution was independently generalized to the midlatitude beta-plane by Yamagata (1976), Tung (1983), and Boyd (1983). In this work, we solve the same problem on the equatorial beta-plane.

In the next section, we briefly review Rossby waves in a shear flow in the middle latitudes. We will later show that many conclusions of the papers mentioned above extend to the tropics. In the following three sections, we discuss our basic equations and the two independent numerical methods we used to solve them. Section 6 analyzes the lack of lateral spreading and the absence of growth in the equatorial solutions. The last part of the paper is a brief summary. 
The main conclusions of Yamagata (1976), Tung (1983), Farrell (1982), and Boyd (1983) are the following:

(1) the Rossby wave spectrum in a linear shear is continuous, not discrete.

(2) The wave energy decays algebraically with time as $1 / t^{2}$ as $t \rightarrow \infty$.

(3) Lines of constant phase rotate clockwise as viewed from above (for shear $S>0$ ).

(4) Latitudinal scale tends to 0 as $t \rightarrow \infty$.

(5) Little latitudinal spreading of the initial disturbance.

(6) A latitudinally-concentrated wavepacket will move north while growing and south while decaying (when $S>0$ ); the southward motion will eventually cease, leaving the packet 'stalled out' at a particular latitude.

(7) A sinusoidal wave tilted against the shear (phase lines running northwest-southeast for $S>0$ ) will amplify for a finite time before entering the asymptotic decay stage.

The second and seventh conclusions are not true for equatorial waves, at least not without important qualifiers. Before trying to explain these surprises, we will first describe our models.

\section{THE PROBLEM: LINEAR SHEAR ON THE EQUATORIAL BETA-PLANE}

The physical model is identical to the model of Boyd and Christidis (1982, 1983): the wave equations linearized about a mean flow $U(y)$ which is a function only of latitude, which allows the separation-of-variables. If we nondimensionalize in such a way that the nondimensional Lamb's parameter is set equal to 1-usual in oceanography, uncommon in meteorology-the equations we must solve are the linearized shallow water wave equations

$u_{t}+i k U(y) u-[y-\mathrm{d} U / \mathrm{d} y] v+i k \phi=0$

$v_{t}+i k U(y) v+y u+\phi_{y}=0$

$\phi_{t}+i k U(y) \phi+i k u+v_{y}=0$

where $k$ is the zonal wavenumber. Note that we always assume a particular zonal wavenumber and a given vertical wavelength (the latter is buried in the nondimensionalization) so that $u, v$, and $\phi$ in (3.1)-(3.3) are functions only of latitude.

We have already discussed the discrete unstable modes of (3.1)-(3.3) in our earlier work. When $U(y)$ has an inflection point, that is, $1-U_{y y}$ changes sign *, the interesting Rossby waves are the barotropically unstable modes,

* Note that $\beta=1$ in our nondimensionalization. 
which are discrete. To explore the continuous spectrum of Rossby waves, and the transient growth phase, we shall therefore confine our attention to barotropically stable mean currents.

All the graphs we shall show here are for the special case of a linear shear

$$
U(y)=S y
$$

where the shear parameter $S$ is a constant. Besides being simple and representative of barotropically stable flows, this will permit comparison with analytic solutions for this same current on the midlatitude beta-plane.

Unfortunately, we have not been able to analytically solve (3.1)-(3.3) even for the particular flow (3.4), so we applied two different numerical methods. The next two sections describe each in turn.

\section{NUMERICAL CALCULATIONS: EIGENVALUE ANALYSIS}

One theoretical observation greatly reduces the amount of computation: equatorial Rossby waves can be increasingly well-modeled by the usual midlatitude beta-plane approximation as the mode number $n$ goes to infinity. One justification is WKB/method-of-multiple-scales reasoning. As $n \rightarrow \infty$, the scale on which the mode is oscillating becomes smaller and smaller. Therefore, the change in the Coriolis parameter between neighboring peaks of the mode becomes smaller and smaller. This in turn implies that the approximation of taking $f$ to be locally constant except where differentiated-the midlatitude beta-plane-becomes more and more accurate. Another argument is more pragmatic: Rossby modes always span the equator, so the midlatitude beta-pane would be a useless approximation unless it was accurate for at least the high order equatorial modes. A more detailed justification of this limiting behavior is given in Boyd (1988).

It follows that it is the low order equatorial Rossby waves which differ most from their midlatitude counterparts. Consequently, it is sufficient to give numerical results only for the lowest symmetric and lowest antisymmetric modes.

Our first set of computations used the same eigensolver as our previous articles, Boyd and Christidis (1982, 1983). We calculated unstable Kelvin waves by deforming the path of integration into the complex $y$-plane to detour around the 'critical latitude' singularity, where the phase speed $c$ is equal to the mean current $U(y)$. To obtain the needed first guess for the iteration, we began with the known solutions for no shear-simple Kelvin and Rossby modes as described in Holton (1975) - and then increased the strength of the shear $S$ in small steps. We then extrapolated a first guess for each new value of $S$ by using the previously computed $c(S)$ for smaller shear. 


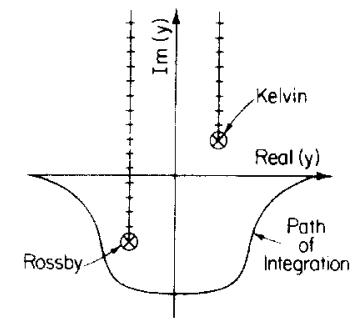

Fig. 1. A schematic of the complex $y$-plane with the path of numerical integration looping below the real axis. (Complex latitudes have no physical meaning, but this detour around the critical latitude singularity makes it possible to track the modes right down to zero mean shear.) The branch cuts from the critical latitude to infinity are also shown for an unstable Kelvin mode and a Rossby mode. Note that the branch cut for the Rossby wave is forced to cut the real axis, implying that the computed solution has a jump discontinuity at that point - and is therefore unphysical. From Boyd and Christidis (1983).

Figure 1 shows what happens when we calculate Rossby and Kelvin waves in linear shear. As the shear increases, the eigenvalue of the Kelvin wave develops a positive imaginary part. This implies that the mode is unstable, but it also legitimizes the choice of complex contour. The critical latitude moves into the upper half of the complex $y$-plane so that we can draw the branch cut from the critical latitude singularity to infinity without crossing either (a) the real y-axis or (b) the contour of integration.

For the Rossby modes, it is a different story. As shown in Fig. 2 of Boyd (1983), $\operatorname{Im}(c)$ for a Rossby mode is negative, shifting the branch point into the lower half-plane. Since the path of integration is also deformed into the lower half-plane, the code implicitly calculates a solution of the differential equations which has the branch cut crossing the real $y$-axis. This follows because the program will necessarily compute a solution which is single-valued on the contour of integration, regardless of whether or not this is the physical solution. If the branch cut does not cross the contour of integration, then Fig. 1 shows that somewhere, it must cut the real axis instead.

Thus, the eigenvalue calculation gives evidence of a negative kind: discrete Rossby modes do not exist in a linear shear flow. This implies that equatorial Rossby modes form a continuous spectrum-just as one can analytically show for their midlatitude counterparts. Instead of an infinite sum over the latitudinal mode number $n$, the Rossby wave component of a general initial-value problem must be expressed as an integral over a continuous phase speed $c$.

5. NUMERICAL CALCULATIONS: INITIAL VALUE ANALYSIS

Since we numerically showed (to no one's surprise!) that a discrete spectrum of Rossby waves does not exist, our second numerical approach 
was to solve the linearized shallow water wave equations as an initial value problem using a fully implicit time-stepping method with fourth order accuracy in $y$. As in the eigenvalue calculation, we assumed the $x$-dependence was sinusoidal with some particular wavenumber $k$.

Initialization is important since the generality of our results depends on the generality of our initial conditions. Since the problem is linear, its general solution is the superposition of the particular solutions obtained by setting the initial condition equal to a given member of a set of complete, orthogonal basis functions. Consequently, letting $\mathbf{u}$ denote the vector with components $(u, v, \phi)$, the general solution to the initial value problem (for a given wavenumber $k$ ) is

$\mathbf{u}_{\text {general }}=\sum_{r=1}^{3} \sum_{m=0}^{\infty} a_{m r} \boldsymbol{\alpha}_{m r}(x, y, t ; k)$

where the $\left\{a_{m r}\right)$ are arbitrary constants and where the initial conditions for the $\alpha$-functions are

$$
\begin{aligned}
\alpha_{m r}(x, y, t=0 ; k)= & \cos (k x) \exp \left(-0.5 y^{2}\right) \times\left\{\delta_{1 r}\left(H_{m}, 0,0\right)^{\mathrm{T}}\right. \\
& +\delta_{2 r}\left(0, H_{m}, 0\right)^{\mathrm{T}}+\delta_{3 r}\left(0,0, H_{m}\right)^{\mathrm{T}}
\end{aligned}
$$

where $\delta_{i j}$ is the usual Kronecker delta, superscript-T denotes the matrix transpose, and $H_{m}(y)$ is the $m$-th Hermite polynomial.

However, solving the linearized wave equations for all possible $\alpha_{m r}$ is very wasteful. First, as explained in the previous section, solutions with small latitudinal scale and broad latitudinal extent will closely mimic the corresponding solutions on the midlatitude beta-plane. Consequently, we should concentrate our attention on small $m$, i.e. the low order Hermite functions.

Second, initial conditions such as (5.2) will excite a mixture of the $m$-th Rossby wave, eastward-travelling gravity wave, and westward-travelling gravity wave. This is rather silly because our previous work (Boyd and Christidis, 1982, 1983) has already studied the behavior of the discrete gravity, mixed Rossby-gravity, and Kelvin waves under shear. Furthermore, these modes are unstable (if the shear is strong enough), so their exponential growth with time will ultimately swamp the algebraic growth or decay of the Rossby waves. What we really want is the most general initial condition that is orthogonal to the gravity and Kelvin modes.

The way to realize this is to use vector Hough-harmonics (Kasahara, 1976) as the basis, and study what happens when the initial condition is one of the low order Rossby Hough functions. As noted in the previous section, there are no discrete Rossby modes in the presence of shear because the Rossby modes form a continuous spectrum (true in spherical geometry as well as the equatorial beta-plane). Nonetheless, the vectors whose compo- 
nents are the zonal velocity, latitudinal current, and height field for meanshear-free equatorial Rossby modes are still members of an orthogonal basis that is complete for all flows on the equatorial beta-plane, linear and nonlinear, stable and unstable. More important, since the high frequency gravity and Kelvin modes are little affected by shears of the strengths used here, the Rossby Hough-harmonics will still be orthogonal (strictly speaking, almost orthogonal) to the Kelvin and gravity modes even in the presence of the mean flow.

Consequently, it is sufficient to study just two cases: the results of initializing with the lowest Rossby Hough-harmonic of each symmetry class, $m=1$ (symmetric about the equator) and $m=2$ (antisymmetric about the equator). For the sake of caution, we made integrations using initial conditions proportional to higher order Rossby Hough-harmonics, too, but the results were similar to those for $m=1,2$, and therefore only these two solutions will be discussed.

The strength of the shear, $S$, and the zonal wavenumber, $k$, remain as parameters. However, the analysis for the midlatitude beta-plane, given in the next section, suggests that growth is largest for small $k$. Although we performed numerical calculations for various $k$, we show only results for the small but otherwise arbitrary case of $k=0.1$ since this is quite representative. The strength of the shear seems to determine only the time scale of the tilt of the phase lines, not the pattern, so we offer graphs only for a single value of $S$.

Figure 2 is a $\log -\log$ plot of the energy as a function of time for two runs that were initialized using the lowest two Rossby Hough-harmonics; the mean current is (2.1) with $S=0.3$. The analytical midlatitude theory predicts that the energy will decay as $1 / t^{2}$ as $t \rightarrow \infty$. The graph shows that the

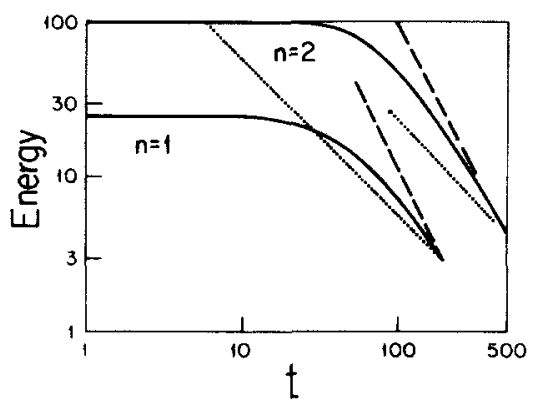

Fig. 2. A $\log -\log$ plot of kinetic energy versus time for runs initialized with the (unsheared) $n=1$ and $n=2$ Rossby modes for zonal wavenumber $k=0.1$. The mean flow $U(y)=0.3 y$. The dotted lines have a slope of $1 / t$; the dashed lines decrease as $1 / t^{2}$. For the rather large range of times shown, the decay of the equatorial waves is intermediate between these two slopes for both modes. 


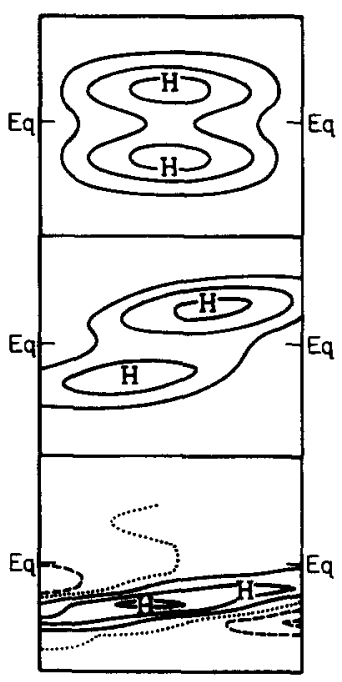

Fig. 3. Isobaric contours, plotted as functions of $x$ and $y$, for three different times: $t=0,30$, and 90. The initial condition was the analytic solution for an $n=1$ Rossby wave for zero shear. The zonal wavenumber $k=0.1$, and the mean current is $U(y)=S y$ where $S=0.3$. Note that for the sake of visual clarity, the $x$-scale is compressed; if both coordinates were plotted on the same scale, the rectangle would be greatly elongated in the $x$-direction. For the same reason, the contour interval was rescaled with time; the wave amplitude at $t=90$ is only a fraction $(\sim 40 \%)$ of its incident value.

rate of decay for equatorial waves is intermediate in slope between $1 / t$ and $1 / t^{2}$ for fairly large times. It is possible that the slope does asymptote to $1 / t^{2}$ for very, very large $t$, but the wave develops finer and finer length scales as $t$ becomes large, so it is difficult to follow the calculation beyond $t=500$ without using thousands of grid points. It is also physically pointless since viscous dissipation will surely become important for such large times *.

It is quite clear, however, that the energy does decay algebraically as $t \rightarrow \infty$. The surprise-interesting but not profoundly important-is that the midlatitude prediction of a $1 / t^{2}$ rate of decrease is misleading.

Figure 3 illustrates the time evolution of the pressure. The graph is strikingly similar to the analogous midlatitude plots given in Boyd (1983). The linear shear tilts the contours of pressure so that the latitudinal scale steadily becomes smaller and smaller.

The wave disturbance drifts southward during the early part of decay and then stalls out - exactly as predicted by the midlatitude analytic theory of Yamagata (1976), who calculated a final latitudinal shift of $-\beta /\left[S\left(k^{2}+\right.\right.$

\footnotetext{
* Haynes (1985) argues that the flow will become unstable for large times as well.
} 
$\left.m^{2}\right)$ ] where $\beta$ is the $y$-derivative of the Coriolis parameter and $m$ is the latitudinal wavenumber. Our initial condition does not generate a simple wavepacket, but the solution can be represented as the superposition of many wavepackets, so the southward drift is hardly surprising.

The one difference from previous midlatitude results which is surprising is the absence of a temporary growth phase. Boyd (1983) has argued that for a midlatitude disturbance, the wave will amplify at the expense of the mean flow for a finite time, perhaps growing by many orders of magnitude if the zonal scale is very large in comparison to the latitudinal scale, before succumbing to the inevitable asymptotic decay. Strictly speaking, this argument only applies to plane waves with an initial tilt against the shear (that is, with lines of constant phase running NW-SE if $\mathrm{d} U / \mathrm{d} y>0$ ), but Boyd (1983, fig. 2) shows that if we superimpose two such components, one tilted against the shear and one tilted with the shear so that their sum is proportional to $\cos (m y)$, the tilted-against wave will still amplify, and may dominate the large time disturbance since the other component is decaying.

There is no sign of this growth in our results, even when we take $k$ very small. (Note that both the length of the period of growth, $m /(k S)$, and the ratio of maximum to initial energy for the tilted-against-the-shear component, $\left(m^{2}+k^{2}\right) / k^{2}$, can be arbitrarily large if the initial latitudinal scale is sufficiently small in comparison to the zonal wavelength.) In the next section, we examine this surprise using the analytic solution which is available for midlatitude Rossby waves to find that they, too, exhibit this same behavior.

6. BROAD WAVEPACKETS OF MIDLATITUDE ROSSBY WAVES: GROWTH AND SPREADING

Our model is the barotropic vorticity equation on the midlatitude betaplane, linearized about a zonal current $U(y)$ which is a function of latitude only

$\psi_{x x t}+\psi_{y y t}+U(y)\left[\psi_{x x x}+\psi_{y y x}\right]+\left[\beta-U_{y y}\right] \psi_{x}=0$

Since our goal is to make comparisons with equatorial waves, we will use the initial condition

$\psi(x, y, t=0) \equiv \mathrm{e}^{i k x} \mathrm{e}^{-(1 / 2) y^{2}} H_{n}(y)$

and restrict attention to the linear profile

$U(y)=S y$ 
The Yamagata-Tung-Boyd solution is most easily expressed using the parameters

$\tau \equiv k S t$

$B \equiv \beta /(k S)$

and the function

$\Phi \equiv \tan ^{-1}[(\tau+m) / k]-\tan ^{-1}(m / k)$

then

$\psi(x, y, t)=(2 \pi)^{-1 / 2} \mathrm{e}^{i k x-i y \tau} \int_{-\infty}^{\infty} \mathrm{d} m \frac{i^{n} \mathrm{e}^{-(1 / 2) m^{2}} H_{n}(m) \mathrm{e}^{-i m y+i B \Phi}\left(k^{2}+m^{2}\right)}{k^{2}+(m+\tau)^{2}}$

The Fourier spectrum of the initial condition is simply

$A(m) \equiv i^{n} \mathrm{e}^{-(1 / 2) m^{2}} H_{n}(m)[t=0]$

since the Hermite functions are (to within the factor of $i$ ) their own transform. This implies that the wave is not a single latitudinal wavenumber as in Boyd (1983), but is rather a wave packet. Furthermore, it is not a spectrum which has a tall, narrow peak about some particular wavenumber $m=m_{0}$, as is required to rigorously justify the wave-tracing arguments of Yamagata (1976), but is rather a disturbance with a broad latitudinal Fourier spectrum.

As the wave evolves in response to the shear flow, the initial Fourier spectrum is modified by two factors. First, the $\exp [i B \Phi]$ represents the usual westward phase propagation of Rossby waves. As noted in the previous section, the beta-effect causes the whole wave disturbance to shift southward (for $S>0$ ) as predicted by Yamagata (1976). Figure 4, which shows the amplitude at $\tau=20$ for three different values of $B$, illustrates this for midlatitude Rossby waves quite clearly. In spite of the fact that the Fourier spectrum is broad instead of narrow, the prediction of Yamagata's midlatitude theory is qualitatively correct. It is not the phase behavior of the equatorial waves which is puzzling; it is their amplitude that is the surprise.

This in turn is controlled by the factor

$$
\Lambda \equiv\left(k^{2}+m^{2}\right) /\left[k^{2}+(m+\tau)^{2}\right]
$$

In the limit $\tau \rightarrow \infty$ for fixed $m$ and $k$, the phase factor $\Phi$ is independent of $\tau$ and $\Lambda \sim\left(k^{2}+m^{2}\right) / \tau^{2}$. The $1 / \tau^{2}$ dependence can be removed from the integral to show that the streamfunction decays algebraically with time for large $t$, and the rate of asymptotic decay is independent of the initial 


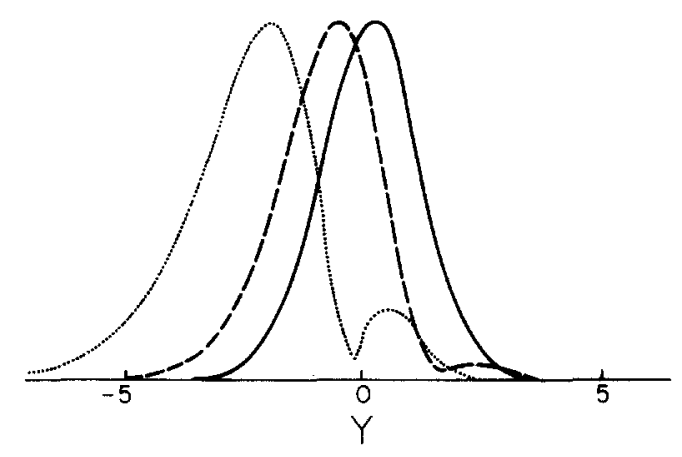

Fig. 4. The absolute value of the amplitude of midlatitude Rossby waves in a linear shear flow for $\tau=20$ and three values of the beta-parameter $B$. The solid curve is $B=0$, the dashes are $B=1$, and the dots trace $B=3$. The initial condition for all three cases was that $\psi$ was such that $\nabla^{2} \psi=\exp \left(i k x-(1 / 2) y^{2}\right)$, i.e. the initial vorticity was a Gaussian in latitude. The peak of the wave packet stops at a latitude farther and farther to the south $(\mathrm{d} U / \mathrm{d} y>0)$ as the beta-effect increases.

condition. As we have already seen, this midlatitude behavior is mimicked by equatorial Rossby waves, and needs no further discussion.

The heart of the mystery lies in the behavior for small time. When $k$ is small, $\Lambda$ grows to a maximum of

$$
\begin{aligned}
\Lambda & \approx\left(k^{2}+m^{2}\right) / k^{2} \\
& \approx m^{2} / k^{2}
\end{aligned}
$$

which is reached at $\tau \approx|m|$. This implies that the contribution of a given wavenumber $m$ will increase to a maximum of $\mathrm{m}^{2} / \mathrm{k}^{2}$ - a very large factor if $k$ is small-before finally succumbing to the $1 / \tau^{2}$ decay for $\tau>|m|$. This is the transient growth phase discussed in Boyd (1983), but as noted above, there is no sign of such growth in the equatorial solutions.

The rub with the reasoning of Boyd (1983) is that it applies only to a single latitudinal wavenumber. Here, however, we have a broad spectrum in latitude, and each wavenumber will undergo its own independent cycle of growth and decay. They key question is not what is happening to a particular wavenumber, but rather: what is happening to the integrated spectrum of waves?

The answer comes from the observation that as $k \rightarrow 0$, the growth factor $\Lambda$ has a very tall, narrow peak centered on $m=-\tau$, and $\Lambda$ tends to a representation of the Dirac delta function, i.e.

$$
\operatorname{limit}_{k \rightarrow 0}\left\{k /\left[k^{2}+(m+\tau)^{2}\right]\right\}=\pi \delta(m+\tau)
$$


This gives the approximation

$\psi(x, y, t) \approx(2 \pi)^{-1 / 2} \pi i^{n} \mathrm{e}^{i k x} \mathrm{e}^{i B \pi / 2} H_{n}(-\tau)\left(\tau^{2} / k\right) \mathrm{e}^{-(1 / 2) \tau^{2}}$

which is valid for

$k \ll \tau$ and $k^{2} \ll \tau^{2} \exp \left[-(1 / 2) \tau^{2}\right]$

$y \sim 0(1)$

What is striking about (6.12) is that far from predicting a transient growth phase for small $k$, it shows that the streamfunction will actually decay exponentially with time. The reason is that for $m$ outside the turning points of the Hermite function, the initial amplitude of the growing wavenumbers is exponentially small. Multiplying an exponentially small amplitude by an algebraically large growth factor gives a tiny result. It is striking that the streamfunction decays rapidly during this stage of moderate time while some components of its spectrum are still growing.

The Hermite function-of-time approximation cannot be uniform in time because for finite $k$, the peak of $\Lambda$ has a finite width and magnitude. Eventually, multiplication by $\left(\mathrm{m}^{2} / \mathrm{k}^{2}\right)$ is no longer large enough to compensate for the smallness of $\exp \left[-(1 / 2) m^{2}\right] H_{n}(m)$. For large times, the wave spectrum is dominated by small and moderate $m$, which decay steadily as $1 / \tau^{2}$.

Overall, the situation is summarized schematically by Fig. 5. The growth

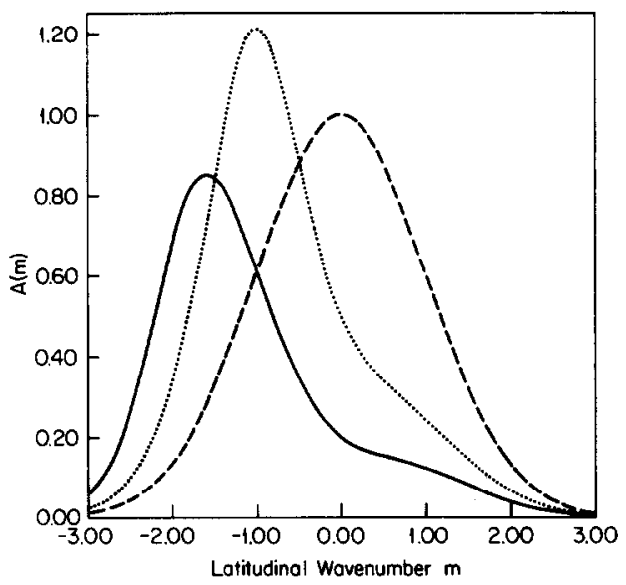

Fig. 5. The latitudinal wavenumber spectrum, $A(m) \Lambda(m, \tau)$, for a midlatitude Rossby wave at three different times: $\tau=0$ (dashed), $\tau=1$ (dotted), and $\tau=2$ (solid curve) for a typical broad initial spectrum. Although part of the spectrum does amplify, the integral $A$ over $m$ decreases monotonically with time, falling from 2.51 to 2.37 to 1.70 for the case shown. 
of large $m$ components is more than compensated for by the decay of small $m$ components so that the energy usually decays even for small times.

Similar conclusions were independently obtained by Shepherd (1985). Although his arguments differ in detail from ours, the physical reasoning is very similar.

Although we have not performed such experiments because of the limitations of our numerical model, there is little doubt that if we superimposed many Rossby modes to create an initial condition orthogonal to the gravity and Kelvin modes, but with a latitudinal Fourier spectrum peaked about some large negative wavenumber, we would observe growth. The point of our work (and Shepherd's) is that unless some forcing mechanism has 'stacked the deck' by creating an initial condition with strong anisotropy, i.e. a few components of large latitudinal wavenumber, then the wave disturbance as a whole will not amplify even though the high latitudinal wavenumber portions, tilted against the shear, may increase by orders of magnitude.

\section{SUMMARY}

We have shown through numerical integration that, unless the mean flow is barotropically unstable, equatorial Rossby waves decay algebraically with time in a shear flow, just like their midlatitude counterparts. Initial conditions in the form of Hermite functions, which describe the usual Rossby modes on a resting mean state, have a broad spectrum in latitudinal wavenumber. Because some wavenumbers $m$ are decaying while others are growing, our numerical solutions show no signs of the transient growth phase discussed in Boyd (1983). We have explained why this is true of both midlatitude and equatorial Rossby waves. In either case, the energy of the wave disturbance will grow a noticeable amount only when the initial condition has a Fourier spectrum in latitudinal wavenumber $m$ which is sharply peaked about some wavenumber $m=m_{0}$ such that $m_{0} \gg k$ where $k$ is the zonal wavenumber of the disturbance, and where the sign of $m_{0}$ is such that the waves are tilted against the mean flow.

Our earlier work has shown that Kelvin and equatorial gravity waves display exotic and complicated behavior at low latitudes. It is refreshing to find that Rossby waves, at least, are not drastically altered by the shift to equatorial latitudes.

\section{ACKNOWLEDGMENTS}

This work was supported by NSF Grants OCE8305648 and OCE8509923. We thank Michael McIntyre for helpful comments and for making Shepherd's (1985) work available to us in advance of publication. 


\section{REFERENCES}

Boyd, J.P., 1978. The effects of latitudinal shear on equatorial waves. Part 1: Theory and methods. J. Atmos. Sci., 35: 2236-2258.

Boyd, J.P., 1983. The continuous spectrum of linear Couette flow with the beta effect. J. Atmos. Sci., 40: 2304-2308.

Boyd, J.P., 1988. Dynamics of the Equatorial Ocean. Springer-Verlag, New York, in press.

Boyd, J.P. and Christidis, Z.D., 1982. Low wavenumber instability on the equatorial betaplane. Geophys. Res. Lett., 9: 769-772.

Boyd, J.P. and Christidis, Z.D., 1983. Instability on the equatorial beta-plane. In: J. Nihoul (Editor), Hydrodynamics of the Equatorial Ocean. Elsevier, Amsterdam, pp. 339-351.

Farrell, B., 1982. The initial growth of disturbances in a baroclinic flow. J. Atmos. Sci., 39: $1663-1686$.

Haynes, P.H., 1985. On the instability of sheared disturbances. J. Fluid Mech., submitted.

Holton, J.R., 1975. The Dynamic Meteorology of the Stratosphere and Mesosphere. Meteorological Monographs, Vol. 15, No. 37. Am. Meteorol. Soc., Boston, 200 pp.

Holton, J.R., 1979. An Introduction to Dynamic Meteorology, 2nd. ed. Academic Press, New York, $390 \mathrm{pp}$.

Kasahara, A., 1976. Numerical integration of the global barotropic primitive equations with Hough harmonic expansions. J. Atmos. Sci., 34: 687-701.

Orr, W. McF., 1907. Stability or instability of the steady motions of a perfect liquid. Proc. R. Irish Acad., 9-69.

Shepherd, T.G., 1985. On the time development of small disturbances to plane Couette flow. J. Atmos. Sci., 42: 1868-1871.

Tung, K.-K., 1983. Initial-value problems for Rossby waves in a shear flow with critical level. J. Fluid. Mech., 133: 443-469.

Yamagata, T., 1976. On trajectories of Rossby wave-packets released in a lateral shear flow. J. Oceangr. Soc. Jpn., 32: 162-168. 